\title{
Star polymers: From conformations to interactions to phase diagrams
}

\author{
C.N.Likos, H.M.Harreis \\ Institut für Theoretische Physik II, Heinrich-Heine-Universität Düsseldorf, \\ Universitätsstraße 1, D-40225 Düsseldorf, Germany
}

Received October 9, 2001

\begin{abstract}
We review recent progress achieved in the theoretical description of the interactions, correlations, and phase behavior of concentrated solutions of star polymers, sterically stabilized colloids, and micelles. We show that the theoretical prediction of an ultrasoft, logarithmically diverging effective interaction between the star centers, which has been confirmed by SANSexperiments and computer simulations, lies in the core of a host of unusual phenomena encountered in such systems. These include anomalous structure factors, reentrant melting behavior, as well as a variety of exotic crystal phases. Extensions to polydisperse stars and the role of many-body forces are also discussed. A particular 'mean-field' character of star polymer fluids is presented and it is shown that it manifests itself in the shape and structure of sedimentation profiles of these systems.
\end{abstract}

Key words: polymers, colloids, liquid-state theory, phase transitions

PACS: 82.70.Dd, 61.25. Hq, 61.20.-p, 64.70.Dv

\section{Introduction}

What are star polymers and why study them? In order to answer this question, we begin from its last part and consider the typical physical systems that are commonly classified under the name 'soft matter'. This term describes a huge variety of substances, the most prominent of which are solutions and suspensions of particles with mesoscopic dimensions, typically $1 \mathrm{~nm}$ to $1 \mu \mathrm{m}$ [1], diffusing into an atomic or molecular solvent. These solute, colloidal particles can have various compositions and molecular architectures. The simplest and most common examples are solid, spherical particles, such as, polystyrene, polymethylmethacrylate (PMMA) or silica spheres, or 'fractal' objects, such as polymer chains. The former are hard and impenetrable, the latter flexible and interpenetrable. Being faced with these two extremes, it is desirable, for both theoretical reasons and practical applications, to design systems that smoothly interpolate between the two, by displaying a tunable 'hardness' in their internal conformations and associated effective interactions [2]. 
Star polymers offer a natural way for bridging this gap. They are macromolecular entities consisting of $f$ polymeric chains chemically anchored to a common center. The number of arms $f$ is also called functionality of the stars. When the degree of polymerization $N$ is the same for all chains, one talks about regular stars. In the limit $N \gg 1$, the microscopic dimensions of the central core where the arms are attached become irrelevant when compared with the overall size of the star and hence we will be making the further simplification of treating the core of the stars as vanishingly small. Related systems are sterically stabilized colloidal particles or micelles, which have star-like behavior at the limit where the size of the core particle becomes much smaller than the extent of the tethered polymeric brush [3-5].

Star polymers have attracted considerable interest in the recent past [6-8]. The reason for that is threefold. First, from a technical point of view, star polymers are important in several industrial applications [6]. One example are hydrogenated polyisoprene star polymers,$^{1}$ which are used as viscosity index modifiers in oil industry applications due to their excellent shear stability. Further, commercial star polymers are brought into action in coating materials, such as binders in toners for copying machines, and in several pharmaceutical and medical applications [6]. Second, from an experimental point of view, the recent synthesis of regular star polymers with various possible numbers of arms by Roovers and coworkers $[9,10]$ made it possible to explore the physics of well-defined model systems, which are monodisperse in both the number of arms and in the degree of polymerization. Important examples are polyisoprene stars with $f=8,18$ [11] and polybutadiene stars with $f=32,64,128$ [10], both synthesized by anionic polymerization. Thereafter, the static properties of stars can be studied by small-angle neutron scattering (SANS), light scattering and small-angle X-ray scattering (SAXS). Quasi-elastic neutron scattering experiments using neutron spin-echo spectroscopy [12], as well as dynamical light scattering are used to study the collective and single-chain dynamics of arms of the star polymers, for a recent review see [6]. Third, star polymers constitute an important soft-condensed matter system, linking the fields of polymer physics and colloid physics, thus attracting interest from a purely theoretical point of view as well. Star polymers with small arm numbers $(f=1,2)$ resemble linear polymers. Thus, their chain-averaged configurations show a considerable asphericity [13-15], although their averaged number density of monomers, $\varphi(r)$, is spherically symmetric around the center of mass of the polymer. With increasing arm number $f$, the asphericity of the stars has been shown to decrease considerably [16-23]. This evolution of the shape of the star with increasing functionality is shown in figure 1 . It is essentially in the limit $f \gg 1$ where a description of star polymers as sterically stabilized colloidal particles holds. This polymer-colloid hybrid character of star polymers has been explored in a number of publications dealing with the structural [4,6,11,24-38] and dynamical $[4,27,28,39-48]$ properties of the same.

The rest of this paper is organized as follows: in section 2 we discuss the conformations of isolated stars and in section 3 we present recent results from theory, experiment, and simulation regarding the effective interaction between them. In sec-

\footnotetext{
1 "Shellvis", distributed by Shell Chemical Co. [6].
} 


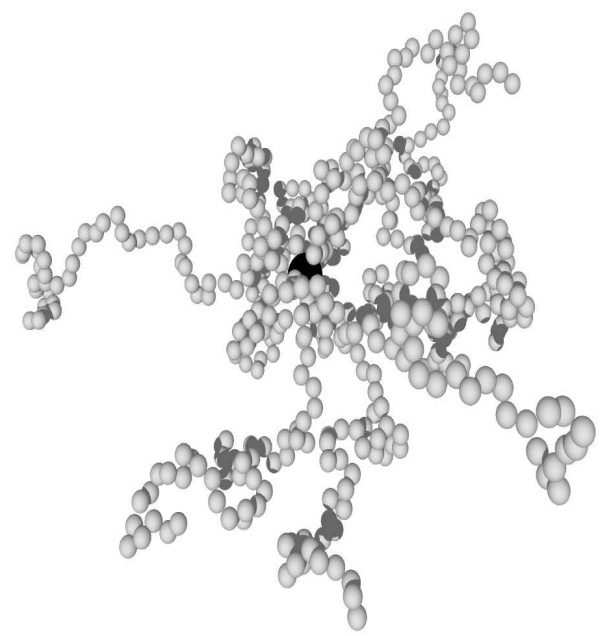

(a)

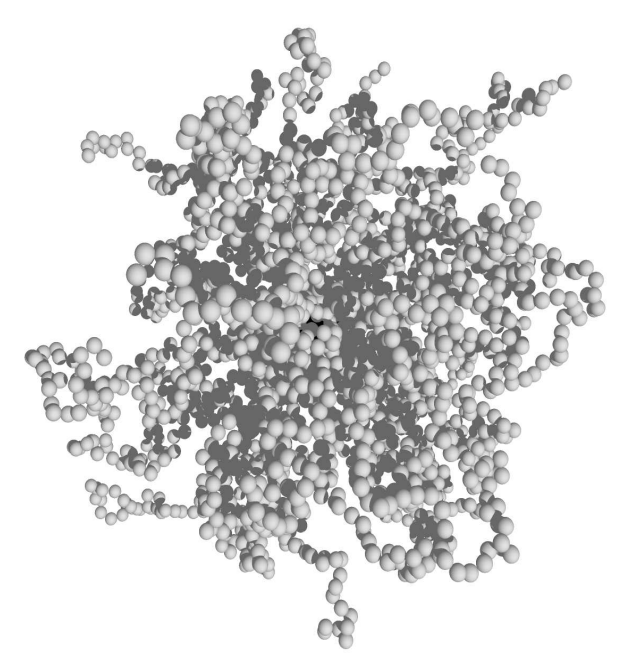

(b)

Figure 1. Snapshots of star polymers in good solvents as obtained from MD simulations employing the model of Grest et al. [49] with: (a) $f=10, N=50$, and (b) $f=50, N=50$. For small $f$, the star looks like a fractal, aspherical object whereas for large $f$ it resembles a spherical, colloidal particle. (Courtesy of A.Jusufi).

tions 5 and 6 we show that star polymer fluids satisfy a certain 'mean-field' limit which manifests itself in the form of their sedimentation profiles. Finally, in section 7 , we summarize and conclude.

\section{Conformations of star polymers}

The polymer chains that are attached on the center of the star undergo constantly thermal fluctuations, akin to those of free polymers in a solvent. The molecular architecture of the macromolecule, however, restricts the freedom of the chains as, on the one hand, the anchored monomer is forced to remain fixed and, on the other hand, many chains are forced to converge at the star center. One expects, therefore, an interplay between three factors: the solvent quality, which determines the value of the excluded-volume parameter $v$ of the chains, the degree of polymerization $N$ and the functionality $f$. Intuitively, it is anticipated that the crowding of $f$ chains in the star interior will cause them to stretch, with the stretching becoming more pronounced as $f$ increases.

Quantitatively, the conformations of single stars are described within the framework of the 'blob model' of Daoud and Cotton [51], schematically depicted in figure 2. In this model, the inner of the star is regarded as a succession of concentric shells of blobs, each blob in the shell having size $\xi(r)$. The spherical blobs are closely packed and within each one every chain behaves as if it were free, i.e., the effects of the neighboring chains are not present. This model yields scaling predictions for the monomer concentration around the star center $\varphi(r)$ and the corona radius $R_{\mathrm{c}}$ of the 
star, defined through the conservation law [51]:

$$
4 \pi \int_{0}^{R_{\mathrm{c}}} \mathrm{d} r r^{2} \varphi(r)=N f
$$

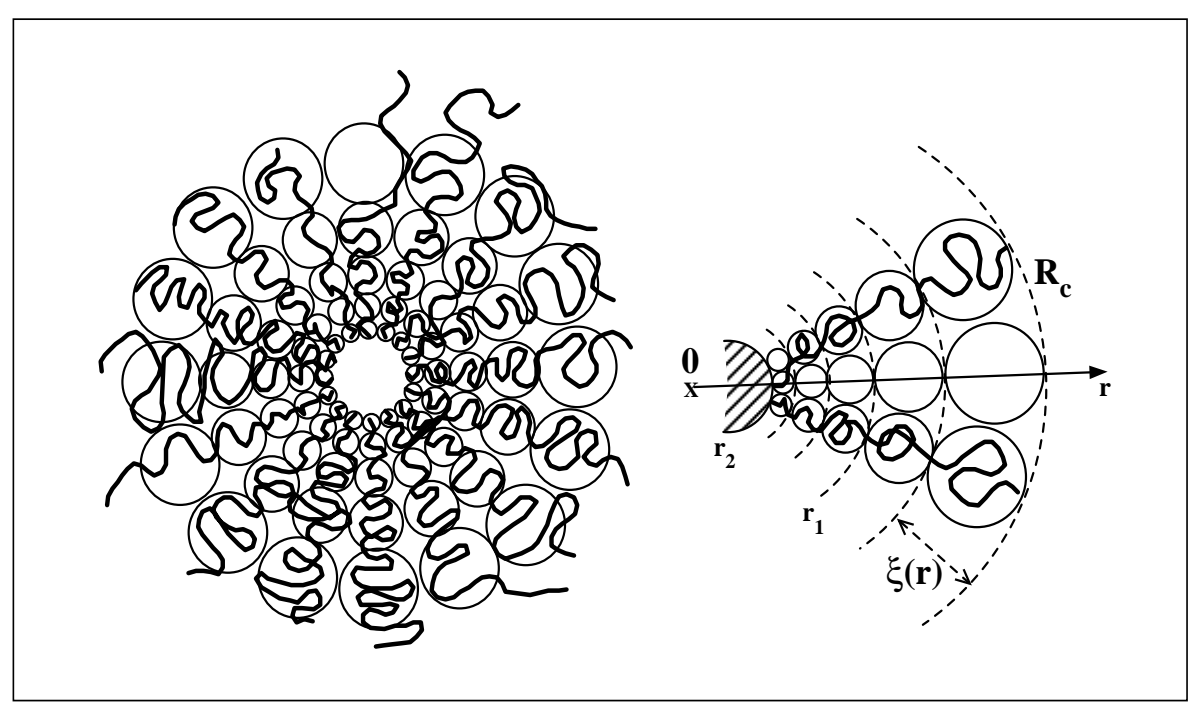

Figure 2. The Daoud-Cotton [51] blob model of a star.

By purely geometrical arguments, it follows that the blob size $\xi(r)$ scales as

$$
\xi(r) \sim r f^{-1 / 2}
$$

Let $n(r)$ be the number of monomers inside a blob centered at $r$. A local swelling parameter $\alpha(r)$ can then be defined as the ratio between the swollen and ideal blob size $\xi_{0}(r)$ :

$$
\alpha(r)=\frac{\xi(r)}{\xi_{0}(r)}
$$

The ideal and swollen sizes are given by $\xi_{0}(r) \sim a n^{1 / 2}(r)$ and $\xi(r) \sim \bar{v} a^{3} n^{3 / 5}(r)$, respectively, yielding the result

$$
\alpha^{5}(r) \sim \bar{v} n^{1 / 2}(r)
$$

where $\bar{v} \equiv v a^{-3}$ is a dimensionless excluded volume parameter and $a$ is the monomer (Kuhn) length. Depending on the value of $\alpha(r)$, one distinguishes three separate regions of the star, namely the following.

I. The swollen region. Here, the blobs are swollen, i.e., their size $\xi(r)$ scales with the number of monomers $n(r)$ with the Flory exponent $3 / 5$, yielding the fol- 
lowing scaling laws for the various quantities of interest:

$$
\begin{aligned}
& n(r) \sim\left(\frac{r}{a}\right)^{5 / 3} \bar{v}^{-1 / 3} f^{-5 / 6}, \\
& \alpha(r) \sim\left(\frac{r}{a}\right)^{1 / 6} f^{-1 / 12} \bar{v}^{1 / 6}, \\
& \varphi(r) \sim a^{-3}\left(\frac{r}{a}\right)^{-4 / 3} \bar{v}^{-1 / 3} f^{2 / 3} .
\end{aligned}
$$

The last equation, combined with the known scaling law for the osmotic pressure $\Pi \sim c^{9 / 4}$ in a semidilute polymer solution [2,52,53], yields the scaling of the local osmotic pressure inside the star as

$$
\Pi(r) \sim f^{3 / 2} r^{-3}
$$

This last equation has been employed by Jusufi et al. [54,55] for the determination of accurate effective potentials acting between star polymers and hard, colloidal particles of arbitrary curvature. ${ }^{2}$

The extent of the swollen region ranges from the outermost of the star, $r=R_{\mathrm{c}}$, down to a distance $r_{1}$ from the center (see figure 2), at which the local scaling factor $\alpha(r)$ attains the unity value. From equation (2.6) we obtain this value as

$$
r_{1} \sim f^{1 / 2} \bar{v}^{-1} a .
$$

II. The unswollen region. The results for the swollen region are based on the assumption that the blob size $\xi(r)$ exceeds the size of the thermal blob $l_{c} \cong a \bar{v}^{-1}$ [52], where the latter is a measure of the distance over which the behavior of the chain is ideal; on longer scales the chain is self-avoiding. However, as $\xi(r)$ becomes smaller with approaching the star center, [see equation (2.2)], at distances $r<r_{1}$ from the center, the blobs have their ideal size, $\alpha(r)=1$, and this leads to the scaling relations:

$$
\begin{aligned}
& n(r) \sim\left(\frac{r}{a}\right)^{2} f^{-1} \\
& \varphi(r) \sim a^{-3}\left(\frac{r}{a}\right)^{-1} f^{1 / 2} .
\end{aligned}
$$

The unswollen region persists down to a distance $r_{2}$ from the center (see figure 2), where the local concentration $\varphi(r)$ reaches the unity value and the star resembles a melt. From equation (2.11) we obtain this value as

$$
r_{2} \sim a f^{1 / 2}
$$

III. The core. At distances $0<r<r_{2}$, the concentration is unity, the blob size coincides with the monomer size and the chains are completely stretched. In this

\footnotetext{
${ }^{2}$ For details see also the article by J.Dzubiella and A.Jusufi in the next issue.
} 
core region of the star there are $N_{c}$ monomers. With $R_{\text {core }}$ denoting the core size, the scaling laws read as

$$
\begin{aligned}
f N_{c} & \sim\left(\frac{r_{2}}{a}\right)^{3}, \\
N_{c} & \sim f^{1 / 2}, \\
R_{\text {core }} & \sim N_{c} a, \\
\varphi(r) & \sim a^{-3} .
\end{aligned}
$$

Combining the results of this section with the definition of the corona radius $R_{\mathrm{c}}$, equation (2.1), the scaling of the latter is obtained as

$$
R_{\mathrm{c}} \sim\left[N f+\frac{1}{10} \frac{f^{3 / 2}}{\bar{v}^{2}}+\frac{1}{6} f^{3 / 2}\right]^{3 / 5} \bar{v}^{1 / 5} f^{-2 / 5} a .
$$

Integrating in equation (2.1) from a lower limit equal to zero, is tantamount to ignoring the size of the microscopic particle on which the chains are attached. Depending on the degree of polymerization $N$ (length of the chains), the strength of the excluded volume interactions $\bar{v}$ and the functionality $f$, one can distinguish three regimes for the $N-, f$ - and $\bar{v}$-dependencies of the star size, as follows:

$$
\begin{aligned}
& \text { Regime } \quad R_{\mathrm{c}} \\
& N \gg f^{1 / 2} \bar{v}^{-2} \rightarrow N^{3 / 5} \bar{v}^{1 / 5} f^{1 / 5} a, \\
& f^{1 / 2} \bar{v}^{-2} \gg N \gg f^{1 / 2} \rightarrow N^{1 / 2} f^{1 / 4} a, \\
& f^{1 / 2} \gg N \rightarrow(N f)^{1 / 3} a \text {. }
\end{aligned}
$$

If the conditions of (2.18) hold, then most of the star is in the swollen region, so that the core and the unswollen part of the star can be ignored. As can be seen from equations (2.18) and (2.19) above, the spatial extension of stars is larger than that of an isolated chain with the same degree of polymerization, due to stretching of the chains caused by the star architecture. At the same time, stars are smaller than a single chain with a degree of polymerization $N f$; the latter has an extent which scales as $f^{3 / 5} N^{3 / 5}$ in good solvents and as $f^{1 / 2} N^{1 / 2}$ in $\Theta$-solvents. The above equations demonstrate that the quality of the solvent alone is not sufficient to determine the scaling behavior of the stars; if the chains are short, then the star can show $\Theta$-type behavior even in a good solvent. In this case, the whole of the star will be in the unswollen region and then the scaling relation (2.19) will be satisfied.

We are going to be considering the case of stars with long chains in a good solvent, so that the swollen region will dominate throughout the extent of the star and the scaling relation (2.18) will hold. Results on isolated star polymers in $\Theta$ solvent conditions can be found in $[6,21-23,39,56,57]$. An effective pair potential describing accurately experimental SANS data for star polymer solutions in the neighborhood of the $\Theta$ point was introduced by Likos et al. [32].

The conformations of isolated stars and the associated predictions of the scaling theory have been tested in a number of microscopic Monte Carlo and Molecular 
Dynamics simulations, both on-lattice $[18,21,23,58-60]$ and off-lattice $[8,19,49,57$, 61-63]. Whereas in on-lattice simulations a coarse-grained model of description is employed automatically, in off-lattice simulations a model microscopic interaction between the beads is used, for a recent review see [60].

A common model for the monomer-monomer interactions in off-lattice simulations in good solvents has been introduced by Grest et al. [49]. All monomers are assumed to interact by means of the purely repulsive truncated and shifted LennardJones (LJ) potential, $V_{0}(r)$, given by

$$
V_{0}(r)= \begin{cases}4 \varepsilon\left[\left(\frac{r}{\sigma_{\mathrm{LJ}}}\right)^{12}-\left(\frac{r}{\sigma_{\mathrm{LJ}}}\right)^{6}+\frac{1}{4}\right] & \text { if } r<2^{1 / 6} \sigma_{\mathrm{LJ}} \\ 0 & \text { if } r>2^{1 / 6} \sigma_{\mathrm{LJ}}\end{cases}
$$

where $\varepsilon$ sets an energy and $\sigma_{\mathrm{LJ}}$ a length scale. Good solvent quality is modeled by a purely repulsive nature of interaction. On the other hand, the connectivity of the chains is modeled by an additional interaction between monomers on the same chain, which is given by the so-called finite extendible nonlinear elastic (FENE) potential $V_{\text {FENE }}(r)$ :

$$
V_{\mathrm{FENE}}(r)= \begin{cases}-15 \varepsilon\left(\frac{R_{0}}{\sigma_{\mathrm{LJ}}}\right)^{2} \ln \left[1-\left(\frac{r}{R_{0}}\right)^{2}\right] & \text { if } r<R_{0}, \\ \infty & \text { if } r>R_{0},\end{cases}
$$

with $R_{0}=1.5 \sigma_{\mathrm{LJ}}$. For small $f, f \lesssim 6$, the interior of the star is not too dense and standard MC simulations are efficient. However, as $f$ increases, it becomes dense and hence one has to employ either MD simulations or local stochastic MC methods $[64,65]$. In addition, it is necessary to attach the chains to a core of microscopic dimensions, so that the problems with overlaps of the innermost monomers can be avoided [63]. In this case, the size of the core has to be taken into account in testing the predictions of the scaling theory regarding the size of the star, as far as in the latter the core size is assumed to be vanishingly small [63]. A detailed study of the chains tethered on a spherical particle of nonnegligible extent can be found in [66].

The scaling law regarding the behavior of the monomer density $\varphi(r)$, equation (2.7), is tested by averaging the latter quantity during the simulation run and plotting $\ln \left[f^{-2 / 3} \varphi(r)\right]$ against $\ln r$. In this way, the exponent $-4 / 3$ has been confirmed in a number of simulations, for a broad range of arm numbers, ranging from $f=3$ up to $f=270$ [6]. The scaling of the star size with $N$ and $f$ is difficult to be verified in a simulation if the corona radius $R_{\mathrm{c}}$ is used as a measure; there always exists a diffuse layer of polymer beyond the scaling regime, whereas in the Daoud-Cotton model an artificially sharp drop of the monomer density to zero at a distance $r=R_{\mathrm{c}}$ from the center is assumed. Therefore, the gyration radius $R_{g}$ is used to characterize the star size. The predictions of the Daoud-Cotton model have been confirmed, both in on-lattice $[18,21,23,58,63,67]$ and off-lattice $[8,22,49,68]$ simulations.

Experimentally, information about the star conformations is obtained by performing scattering from very dilute solutions, where the effects of star-star interactions are vanishingly small. In this way, the only contribution to scattering is 
the form factor $P(Q)$ of the stars, which is related to the Fourier transform of the monomer density $\varphi(r)[6,69,70]$. A common and successful fit for the form factor, which is consistent with the predictions of the Daoud-Cotton theory, is provided by the Dozier expression [11]:

$$
V_{W} P(Q)=V_{W} \exp \left[-\frac{\left(Q R_{g}\right)^{2}}{3}\right]+\frac{4 \pi \alpha}{Q \xi} \frac{\sin \left[\mu \tan ^{-1}(Q \xi)\right]}{\left[1+(Q \xi)^{2}\right]^{\mu / 2}} \Gamma(\mu),
$$

where $V_{W}$ denotes the molar volume, $\mu=1 / \nu-1, \xi$ is the average blob size, $\alpha \sim \bar{v}(\xi / a)^{1 / \nu}$ and $\Gamma(\mu)$ is the gamma function. According to Dozier et al. [11], the quantities $V_{W}, \alpha, \xi$ and $R_{g}$ are to be treated as free fit parameters, whereas the exponent $\nu$ is fixed at its Flory value $3 / 5$, yielding $\mu=2 / 3$. The Dozier form factor yielded excellent fits for an array of data obtained for stars with $f=8-128$ arms [30].

\section{Effective interactions between star polymers}

We now consider a concentrated solution of $N_{s}$ star polymers enclosed in a macroscopic volume $V$. The questions that arise are what kinds of spatial correlations, structure and phases will be displayed by this system, as a function of the concentration, functionality, and temperature. To simplify matters, we focus now on the case of athermal solvents, for which temperature is an irrelevant thermodynamic parameter, as the monomer-monomer interactions are purely of the excluded-volume type. Hence, no independent energy scale appears in the problem other than the thermal energy $k_{\mathrm{B}} T$.

A powerful idea to analyze such complex fluids is that of introducing an effective interaction $v(r)$ between the stars, which formally results in canonically tracing out all monomer degrees of freedom while keeping their centers held at a distance $r$ apart [2]. Once such an effective interaction has been derived, it is then possible to treat the complicated star-polymer fluid in a drastically simplified fashion: the stars become 'point particles' interacting via the potential $v(r)$ and the known techniques from liquid-state theory can be employed in order to quantitatively describe the fluid [71]. It can be shown that the process of 'partial elimination' of degrees of freedom, which is employed in the derivation of the effective interaction, exactly preserves both the overall thermodynamics and the correlations of the star centers in the effective picture [2].

\subsection{Theory}

Formally, the effective potential between the star centers (considered for simplicity to be point particles featuring no direct interaction with one another) is proportional to the logarithm of the ratio of the partition function $Q_{2}(r)$ of two stars at distance $r$ over the product of the two partition functions of the isolated stars, $Q_{1}^{2}[2]$ :

$$
\beta v(r)=-\ln \left[\frac{Q_{2}(r)}{Q_{1}^{2}}\right]
$$


where $\beta \equiv\left(k_{\mathrm{B}} T\right)^{-1}$.

The pioneering work in establishing scaling relations for the ratio on the rhs of equation (3.1) above was carried out by Witten and Pincus [25]. Consider first the case of single chains $(f=1)$, for which the 'center' has to be interpreted as the end-monomer (alternatively one can look at single chains as 'stars' with $f=2$ if one chooses the central monomer to be the effective degree of freedom.) It is known that the partition function $Q_{1}$ of a single chain is a power-law of the number of monomers $N[52]$ :

$$
Q_{1} \propto \tau^{N} N^{\gamma-1}
$$

where $\gamma=7 / 6$ is a universal exponent, whereas $\tau$ is a parameter the value of which depends on the microscopic details of the chain. Let us consider the case where $r$ is of the order of the monomer length, $a$. Then, the bulk of the system will look like a single fluctuating chain with $2 N$ monomers. Accordingly, the partition function $Q_{2}(r \sim a)$ will be given by the general result, equation (3.2), with $N \rightarrow 2 N$, namely

$$
Q_{2}(r \sim a) \propto \tau^{2 N}(2 N)^{\gamma-1}
$$

Equations (3.2) and (3.3) yield

$$
\frac{Q_{2}(r \sim a)}{Q_{1}^{2}} \propto N^{1-\gamma}
$$

When the chains are looked upon at scales where the microscopic details are not visible, the ratio of the partition functions can only depend on the dimensionless ratio $r / R$, where $R$ denotes the typical chain size. ${ }^{3}$ At the same time, it is a power law in $N$ and the latter enters the combination $r / R$ only through the dependence of the chain size on the degree of polymerization, $R \propto N^{\nu}$. The consequence is that $Q_{2}(r \sim a) / Q_{1}^{2}$ must be a power law in $r / R$,

$$
\frac{Q_{2}(r \sim a)}{Q_{1}^{2}} \propto\left(\frac{r}{R}\right)^{x} \propto N^{-x \nu}
$$

Equations (3.4) and (3.5) imply $x=(\gamma-1) / \nu$. Though the derivation is based on the assumption $r \sim a$, the result of equation (3.5) can be extended up to $r \sim R$. Indeed, at these distances, one expects the effective interaction to have fallen practically to zero, as the two chains do not overlap, and equation (3.5) yields $Q_{2}(r \sim R) / Q_{1}^{2}=1$ there, with the implication that $\beta v(r)$ from equation (3.1) vanishes. The scaling argument yields the correct physical behavior at both extreme cases, $r \sim a$ and $r \sim R$, hence we can write, using equation (3.1),

$$
\beta v(r)=-\frac{\gamma-1}{\nu} \ln \left(\frac{r}{R}\right) \quad(r \leqslant R) .
$$

Note the ultrasoft, logarithmic divergence of the effective interaction as $r \rightarrow 0$.

\footnotetext{
${ }^{3}$ It is at this point irrelevant whether $R$ stands for the gyration radius, the end-to-end radius or the corona radius, since all these quantities simply differ by factors of order unity.
} 
The extension of these ideas to large functionalities $f$ is straightforward and is based on geometrical considerations of counting the number of Daoud-Cotton blobs in a star [25]. The main result is the generalization of equation (3.2) to arbitrary $f$ which reads as:

$$
Q_{1} \propto\left[N f^{(\nu-1) /(2 \nu)}\right]^{-\nu f^{3 / 2}}
$$

Following the same steps as for the case $f=1$ we obtain

$$
\beta v(r)=-\alpha f^{3 / 2} \ln \left(\frac{r}{R}\right) \quad(r \leqslant R),
$$

with the unknown proportionality factor $\alpha$. The logarithmic divergence is recovered again.

Likos et al. [31], introduced thereafter a complete effective interaction potential between star centers for arbitrary $f$. By matching the expression (3.6), valid for $f=1$, with the expression (3.8), valid for $f \gg 1$, it was postulated that $\alpha=$ $(\gamma-1) / \nu=5 / 18$ for all $f$. This assumption evidently gives the exact result for $f=1$. For $f=2$, it yields for the prefactor $\alpha f^{3 / 2}$ the value 0.786 , which is very close to the exact value 0.8 calculated by means of renormalization group techniques by des Cloizeaux [72]. Moreover, recent renormalization group calculations show that the functional form $(5 / 18) f^{3 / 2}$ yields excellent agreement with the 'exact' value, at least up to $f=6[73-76]$.

The logarithmic interaction was assumed to set in whenever significant overlap between the blobs of the two stars takes place. Thereafter, the length scale $R$ was fixed as the length at which the outermost blobs of the two stars fully overlap. Hence, by defining $\sigma / 2$ as the distance from the star center to the center of the outermost blob (see figure 2), the logarithmic interaction was assumed to be valid for $r \leqslant \sigma$. For distances $r \geqslant \sigma$, the interaction was postulated to have a Yukawa form, with the decay length given by the diameter of the outermost blob, $\xi_{\max }=2 \sigma / \sqrt{f}$, as dictated by geometry. This is intuitively clear, as the blob size is the only relevant length scale at weak overlaps between the chains. This kind of decay is valid only for sufficiently large functionalities, $f \gtrsim 10$, for which the blob picture applies. For small functionalities, Jusufi et al. [54] established that the Yukawa decay must be replaced by a Gaussian decay.

By combining the logarithmic with the Yukawa form and matching them at $r=\sigma$ by requiring that both the potential and its first derivative (the force) be continuous there, the effective star-star interaction was put forward [31]. For $f \gtrsim 10$, it reads as:

$$
\begin{aligned}
\beta v(r) & =\frac{5}{18} f^{3 / 2}\left[-\ln \left(\frac{r}{\sigma}\right)+\frac{1}{1+\sqrt{f} / 2}\right] & & \text { for } r \leqslant \sigma, \\
& =\frac{5}{18} f^{3 / 2} \frac{1}{1+\sqrt{f} / 2}\left(\frac{\sigma}{r}\right) \exp \left[-\frac{\sqrt{f}(r-\sigma)}{2 \sigma}\right] & & \text { for } r \geqslant \sigma .
\end{aligned}
$$

Comparisons with simulations established that $\sigma \cong 1.32 R_{g}$ [63]. The potential is shown in figure 3 for various values of the functionality $f$. It can be seen that it 


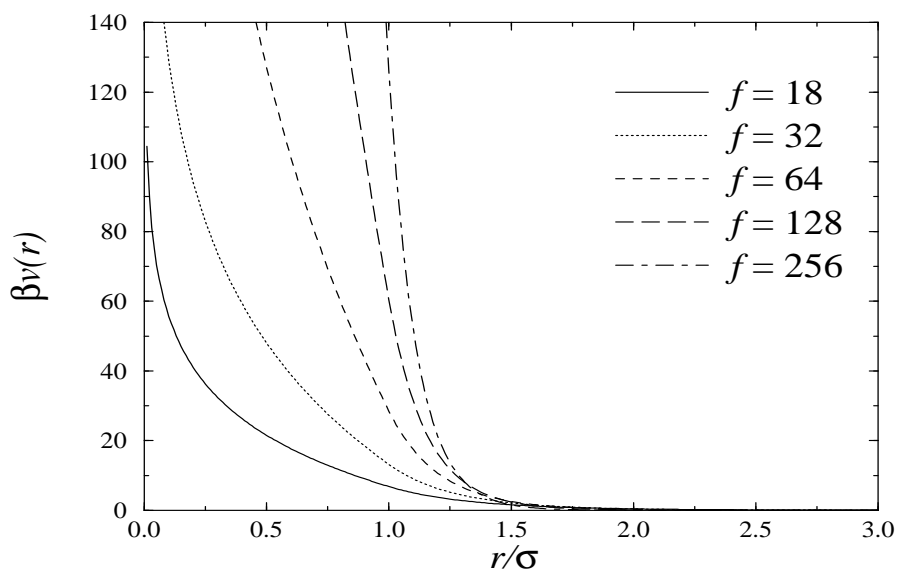

Figure 3. The effective star-star potential of equation (3.9) for various $f$-values.

becomes harder with increasing $f$, tending eventually to a hard-sphere interaction which formally obtains in the limit $f \rightarrow \infty$.

The effective potential valid for $f \lesssim 10$ has the form [54]:

$$
\begin{aligned}
\beta v(r) & =\frac{5}{18} f^{3 / 2}\left[-\ln \left(\frac{r}{\sigma}\right)+\frac{1}{2 \tau^{2} \sigma^{2}}\right] & & \text { for } r \leqslant \sigma, \\
& =\frac{5}{18} f^{3 / 2}\left[\frac{1}{2 \tau^{2} \sigma^{2}} \exp \left[-\tau^{2}\left(r^{2}-\sigma^{2}\right)\right]\right] & & \text { for } r \geqslant \sigma .
\end{aligned}
$$

Here, $\sigma$ and $\tau(f)$ were determined by comparison to simulation. Again, it turns out that $\sigma \cong 1.32 R_{g}$ and $\tau(f)$ is of the order of $1 / R_{g}$ and is obtained by fitting to computer simulation results. For $f=2$, the value $\tau \sigma=1.03$ was obtained, which, together with the potential in equation (3.10) above, yields, for the second virial coefficient of polymer solutions, the value $B_{2} / R_{g}^{3}=5.59$, in agreement with the estimate $5.5<B_{2} / R_{g}^{3}<5.9$ from renormalization group and simulations [77]. For $f=5$, it was found that $\tau \sigma=1.12$, which leads to $B_{2} / R_{g}^{3}=11.48$, in accordance with Monte Carlo simulation results [78,79].

\subsection{Simulations}

Jusufi et al. [63] performed extensive Molecular Dynamics simulations for a large variety of combinations of arm numbers and degrees of polymerization of the stars. The simulations involved two star polymers, the centers of which were kept fixed at a distance $r$, while the monomeric degrees of freedom in the arms were moved. The monomer-monomer interactions used were the truncated and shifted LennardJones potential of equation (2.21) between all monomers and, in addition, the FENE potential of equation (2.22) for monomers along the same chain. Due to the large number of arms $f$, which could not be accommodated in the small region at the center of the star $[49,57]$, the arms had to be attached on a spherical particle of microscopic size $R_{d} \sim \sigma_{\mathrm{LJ}}$. 

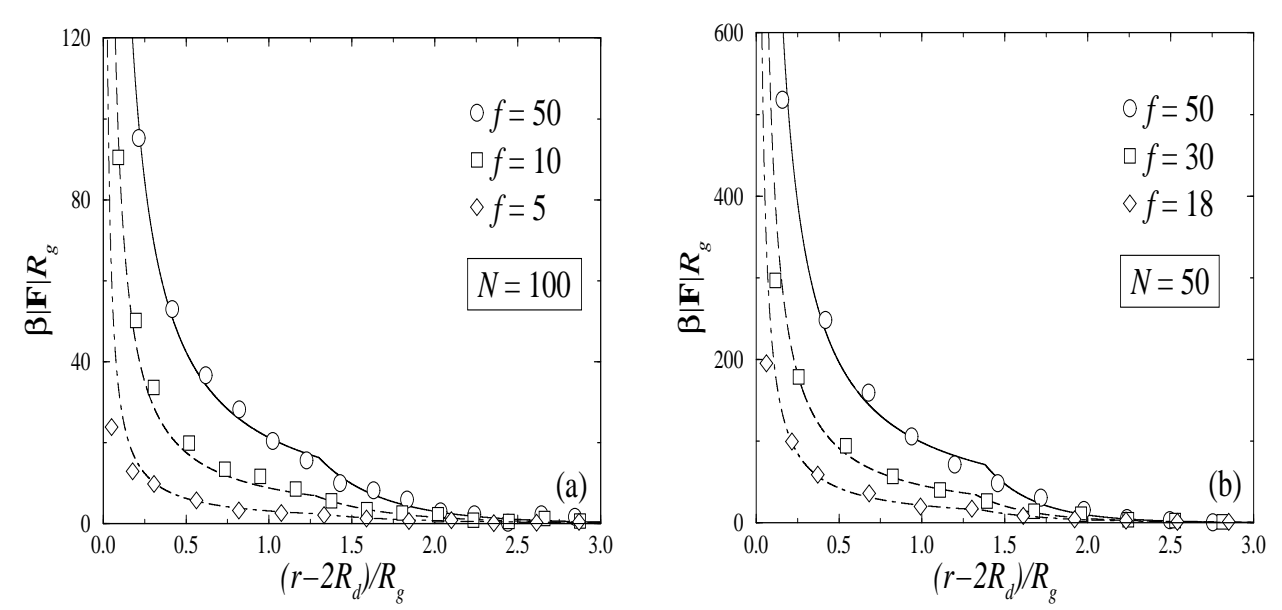

Figure 4. Simulation results (symbols) and the theoretical prediction (lines) for the magnitude of the reduced effective force $\beta|\mathbf{F}| R_{g}$ between the centers of two star polymers, plotted against the normalized distance $\left(r-2 R_{d}\right) / R_{g}$.

The results are shown in figure 4, where an excellent agreement between theory and simulation can be seen. ${ }^{4}$ It should be noted that the kink in the theoretical force curves appearing at $r=\sigma$ stems from the fact that, by construction, the potential is continuous there only up to its first derivative. The second derivative of $\beta v(r)$ has a jump there, however, and this shows up as a kink in the force vs. distance curves. Moreover, the Yukawa tail of the potential was carefully checked by plotting the logarithm of the force against distance for $r>\sigma$ [63] and finding that the simulation data fell on a straight line, the slope of which was given by the decay length of the effective potential of equation (3.9).

Further Monte Carlo simulations were carried out by Rubio and Freire [79] for star polymers of functionalities $f=4-18$. In this work, the second virial coefficient was measured and compared with the one predicted from the potential of equation (3.9). Discrepancies at large star separations were found, which have a noticeable effect on the second virial coefficient but not on the distribution functions. However, in view of the fact that the functionalities simulated in [79] were small, it is not a surprise that the potential of equation (3.9) turns out to be inaccurate for large separations: the Yukawa decay of this potential should be replaced with a Gaussian decay, and the resulting, modified interaction of equation (3.10) indeed yields the correct values of the second virial coefficients. Shida et al. [80] recently performed monomer-resolved Monte Carlo simulations and measured the radial distribution function $g(r)$ of two stars, which is directly connected to the "potential of mean force' $v(r)$ via $g(r)=\exp [-\beta v(r)]$. The results of these simulations matched

\footnotetext{
${ }^{4}$ In this article, we focus our attention on the case $f \gtrsim 10$, for which the logarithmic-Yukawa potential of equation (3.9) is valid, because it is for such functionalities that we obtain several phase transitions for the system. For small $f$, the system is in the fluid state for all densities, as will be shown in section 4 . For a comparison between theory and simulation regarding the potential of equation (3.10), see [54].
} 
very well with the theory for the values $f=6,12,18$, and 24 studied there. Further corroboration for the validity of the interaction was offered by the recent work of Striolo et al. [81].

\subsection{Experiments}

A detailed comparison between theory and SANS data from a series of solutions of 18-arm polyisoprene (PI) stars was performed by Likos et al. [31], and for 57-arm solutions by Stellbrink et al. [82]. In [31], samples with volume fractions $\phi$ in the range $5 \cdot 10^{-4} \leqslant \phi \leqslant 0.3$ were investigated. The theoretically obtained structure factor of the stars, calculated by employing the potential of equation (3.9), was multiplied by the form factor of the stars and convoluted with the known resolution function of the experimental apparatus in order to obtain the theoretical prediction for the total scattering intensity $I(Q)$ at momentum transfer $Q$. The star size was taken from the experiment and no adjustable parameters were used. This was then compared to the measured intensities. The results are shown in figure 5.
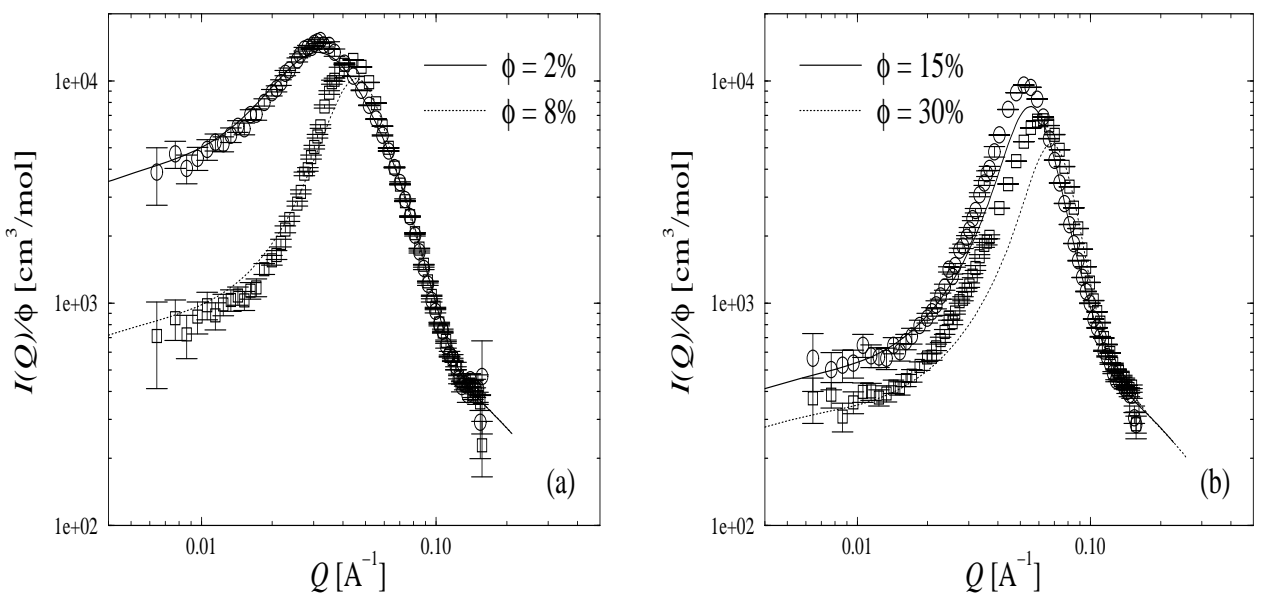

Figure 5. Experimental (symbols) and theoretical (lines) results for the scattering intensities $I(Q)$ scaled with polymer volume fraction $\phi$. From top to bottom (a) $\phi=2 \%$ and $8 \%$; (b) $\phi=15 \%$ and $30 \%$. The overlap volume fraction is $\phi_{*}=10 \%$ for this sample. (Redrawn from [31].)

It can be seen that the agreement is quite satisfactory for the whole range of concentrations. In particular, the osmotic compressibility of the solution, being proportional to $I(Q \rightarrow 0)$ is given correctly for all concentrations, as well as the general shape and wavenumber $Q_{\max }$ at which the scattering intensity displays a maximum. The height of the peak is underestimated by the theory, and the agreement worsens somewhat as the concentration grows. However, at high values of $\phi$, the decoupling between form- and structure factors implied in writing down $I(Q) \propto P(Q) S(Q)$ becomes questionable and this is a possible source of discrepancies between theory and experiment. The logarithmic-Yukawa potential is the first that gave quantitative agreement between theory and experiment for such a wide range of concentrations. 
Earlier attempts to fit the experimental results with a hard sphere-Yukawa interaction, for example, failed at and beyond the overlap concentration [83,84]. Indeed, the existence of a 'soft core', such as the logarithmic term in the potential is crucial at high concentrations where the stars start interpenetrating. A similar comparison performed recently for $f=57$-arm samples again yielded a very satisfying agreement between theory and experiment [82]. Further experimental support for the interaction potential is offered by shear moduli measurements on micelles [85] and osmotic second virial coefficient measurements for star polysterenes [81].

\subsection{Polydispersity effects and many-body forces}

The chemical synthesis of monodisperse chains, i.e., of chains with a fixed number of monomers $N$ is nowadays relatively straightforward. However, experimental star polymer samples display inevitably polydispersity in the arm number $f$ which reflects itself into a size polydispersity by means of equation (2.18) as well. The distribution of arm numbers of a sample around its mean value is rather sharp for arm numbers $f \cong 30$ but grows with the increasing $f$. Typical values of the relative polydispersity are of the order of $5 \%$ for $f \cong 60$. Hence, it is desirable to extend the theory of effective interactions to polydisperse samples. This was done by von Ferber et al. [37], using a combination of scaling ideas and computer simulations. It was established that the effective interaction $v_{f_{1} f_{2}}(r)$ between two stars of functionalities $f_{1}, f_{2}$ and respective sizes $\sigma_{f_{1}}, \sigma_{f_{2}}$ is given by the form [37]:

$$
\begin{aligned}
\beta v_{f_{1} f_{2}}(r) & =\Theta_{f_{1} f_{2}}\left[-\ln \left(\frac{r}{\sigma}\right)+\frac{1}{1+\kappa \sigma}\right] & & \text { for } r \leqslant \sigma, \\
& =\Theta_{f_{1} f_{2}} \frac{1}{1+\kappa \sigma}\left(\frac{\sigma}{r}\right) \exp [-\kappa(r-\sigma)] & & \text { for } r \geqslant \sigma,
\end{aligned}
$$

with the scaling prefactor

$$
\Theta_{f_{1} f_{2}}=\frac{5}{36} \frac{1}{\sqrt{2}-1}\left[\left(f_{1}+f_{2}\right)^{3 / 2}-\left(f_{1}^{3 / 2}+f_{2}^{3 / 2}\right)\right]
$$

and the mean size $\sigma$ and decay length $\kappa$

$$
\sigma=\frac{\sigma_{f_{1}}+\sigma_{f_{2}}}{2}, \quad \kappa^{-1}=\frac{\kappa_{f_{1}}^{-1}+\kappa_{f_{2}}^{-1}}{2} .
$$

Here $\kappa_{f_{i}}^{-1}=2 \sigma_{f_{i}} / \sqrt{f_{i}}$ is the decay length of the monodisperse interaction, equation $(3.9)$.

On the other hand, the process of tracing out the monomers in order to derive effective interactions between the star centers always has the effect of generating a hierarchy of forces, commencing at pair interactions and proceeding to include triplet, quadruplet and higher-order terms [86]. It is usual practice to truncate this series at the pair level, introducing thereby solely pair interactions, such as the ones we have presented in the preceding subsections. Nevertheless, the missing higher-order terms should be estimated, in order to establish the validity of the pair potential 
approximation. For stars, this was done by von Ferber et al. [36]. As a first result, it was found that triplet forces become relevant only when three stars start to have triple overlaps within their coronae, yielding a packing fraction which is much higher than the highest shown in figure 6 . Hence, the star polymer phase diagram shown there remains unaffected by the presence of three-body forces. Moreover, the triplet force was found to be attractive, i.e., the total force turns out to be smaller than the sum of the two body forces. This can be understood intuitively, if one imagines that the force arises from an overlap between the stars' coronae. In a geometry with a triple overlap, the sum of the three two-body overlaps overestimates the total overlap volume, as it counts the triplet overlap three times. On a quantitative basis, it was found that independently of the arm numbers and of $r$, in the full region described by the logarithmic potential, the relative deviation of the force caused by the triplet forces is equal to $\left(3^{3 / 2}-3\right) /\left(2^{3 / 2}-2\right) \cong-0.11$ [36]. At very high concentrations, where the system looks like a semidilute polymer solution, the pair potential approximation breaks down, however. This was established in the work of von Ferber et al. [36], through the investigation of the general $M$-body forces in the limit when the interstar distances go to zero. It was found that the contribution from a cluster of $M$ stars increases with $M$ and even diverges for $M \rightarrow \infty$.

\section{The phase diagram of star polymer solutions}

Taking the pair potential of equation (3.9) for granted, it is then possible to trace out in detail the phase diagram of the system. Clearly, as the temperature plays no role, the relevant parameters are the functionality $f$ and the density $\rho=$ $N_{s} / V$ of stars, which was expressed through the dimensionless 'packing fraction' $\eta=(\pi / 6) \rho \sigma^{3}$. The phase diagram was calculated by Watzlawek et al. $[35,38]$.

For the fluid phase, the very accurate and thermodynamically consistent RogersYoung closure [87] was solved to obtain pair correlations and structure factors. The free energy of the fluid phase was then calculated by means of the compressibility or virial routes [71]. In order to measure the fluid free energy in a simulation, the technique of thermodynamic integration was employed [88,89].

As far as the solid phases are concerned, Watzlawek et al. [35,38] considered a large number of candidate crystal structures and the competition among them. The structures were the fcc, bcc and simple cubic (sc) lattices, as well as the more 'exotic' diamond (diam), face-centered orthogonal (fco), body-centered orthogonal (bco), orthogonal (orth) and diamond orthogonal (diao) structures [90]. In the last four, 'orthogonal' structures, produced by stretching the conventional unit cells of the original ones by an arbitrary amount and keeping the conventional cell volume fixed, the ratios of the lattice constants $a, b$ and $c$ in the three spatial directions act as additional degrees of freedom which have to be relaxed so that a minimum of the free energy can be achieved. ${ }^{5}$ The analysis was carried out for a wide range of arm

\footnotetext{
${ }^{5}$ We note that the bco structure reduces to the bcc for $a: b: c=1: 1: 1$ and to the fcc for $a: b: c=1: 1: \sqrt{2}[90]$.
} 
numbers, $18 \leqslant f \leqslant 512$ and packing fractions, $0 \leqslant \eta \leqslant 1.5$. The common tangent construction yielded then the phase diagram shown in figure 6 .

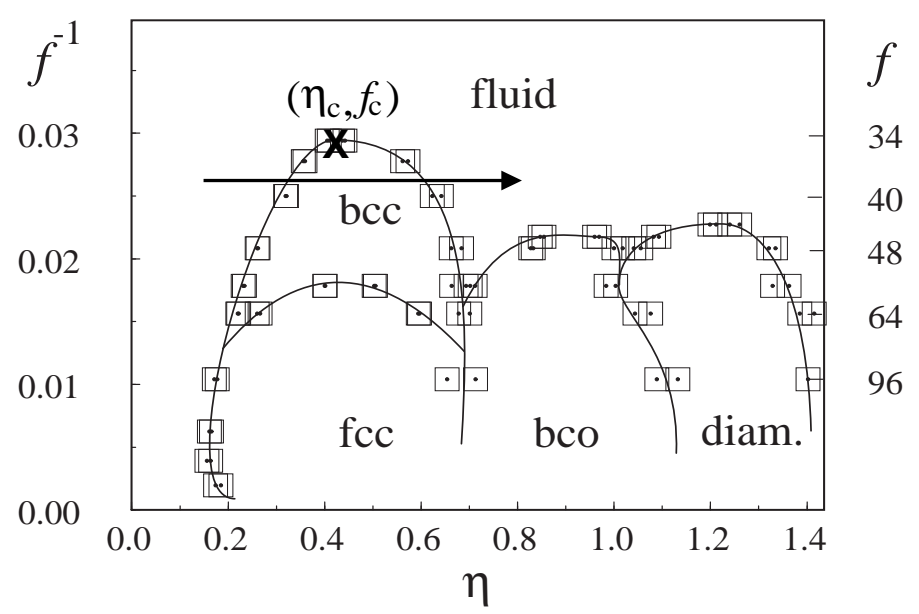

Figure 6. Bulk phase diagram of star polymers interacting with potential of equation (3.9), calculated in [35]. The squares indicate the phase boundaries; solid lines are guide to the eye. The black cross denotes the point with critical arm number $f_{\mathrm{c}} \simeq 34$ and corresponding density $\eta_{\mathrm{c}} \simeq 0.43$. The arrow indicates a path through the phase diagram that is roughly equivalent to a change in the altitude $z$ when the solution is under the influence of a gravitational field, see section 6 .

For $f \lesssim f_{\mathrm{c}}=34$, the fluid phase is the only stable one and freezing is impossible at all densities. We note that this feature had been predicted by Witten et al. [24] using scaling arguments. These authors had estimated $f_{\mathrm{c}} \cong 100$. The detailed phase diagram also confirms the reentrant melting scenario put forward in [24], with the bcc crystal being nested between a low- and a high-density liquid at about the overlap packing fraction $\eta_{*} \cong 0.50$. For $f \geqslant f_{\mathrm{c}}$, four stable crystal structures exist and, depending on $f$, a given system will have at least one stable crystalline phase. In the domain of intermediate densities, $0.2 \lesssim \eta \lesssim 0.7$, the bcc phase is stable for $f_{\mathrm{c}}<f \lesssim$ 54 , whereas for $f \gtrsim 70$ the fcc crystal wins. In the regime $54 \lesssim f \lesssim 70$, the system will first freeze into a bcc lattice but then it will undergo a structural phase transition into a fcc crystal. The interplay between bcc and fcc can be easily understood here, as at these densities only the Yukawa tail of the interaction is visible to the particles. Hence, for large $f$ (strong screening), packing effects dominate and stabilize the fcc, whereas for smaller $f$, energy plays an important role stabilizing the more open, bcc structure. This is completely analogous to the phase behavior observed in chargestabilized colloids [91-93] as well as in copolymer micelles [4,5,94,95]. The HS-type behavior, formally corresponding to $f \rightarrow \infty$ sets in at very high $f$-values, typically $f \cong 10000$, due to an interplay between the steeply increasing logarithmic core and the Yukawa tail, for details see [38].

Quite more unexpected phases show up for high packing fractions, $\eta \gtrsim 0.7$. A bco phase with a strong anisotropy along its three principal space axes is stable in the 
region $0.70 \lesssim \eta \lesssim 1.1$ and a diamond lattice becomes stable for $1.1 \lesssim \eta \lesssim 1.4$. The ratios of the lattice constants in the bco crystal turned out to be nearly independent of $f$ and increasing from $b / a \cong 2.24$ and $c / a \cong 1.32$ at $\eta=0.7$ to $b / a \cong 3.14$ and $c / a \cong 1.81$ at $\eta=1.0$. This means that, throughout the range of stability of the bco phase, the anisotropy in the conventional unit cells leads to a strong interpenetration of the particle coronae along one of the three lattice axes (the one with the smallest lattice constant). In this way, the nearest-neighbor distance is smaller than $\sigma$ whereas all other neighbors are kept at distances larger than $\sigma$. This can be intuitively understood from the form of the interaction potential (3.9). Due to the very weak divergence at small $r$, the energy penalty for overlaps within the logarithmic core is not prohibitively large. At the same time, the potential falls rapidly enough at distances $r>\sigma$, so that it becomes energetically favorable to have two neighbors very close to a central particle and all remaining ones outside the $\sigma$-range rather than twelve (fcc) or eight (bcc) nearest neighbors, all of them at distances larger than the nearest neighbor distance in the bco but nevertheless within the $\sigma$-range.

The same argumentation leads to an understanding of the stability of the diamond lattice. As the density increases further, the bco becomes unstable with respect to a lattice in which two more neighbors enter into the corona and the diamond lattice with four nearest neighbors is stabilized. Again, the simulations revealed that in the domain of stability of the diamond, the second neighbors are kept outside the corona. Hence, both the ultrasoft character of the logarithmic potential and the crossover to a Yukawa tail at $r=\sigma$ are necessary to stabilize the diamond crystal. This phenomenon is unknown for the usual, hard pair potentials and, in fact, there exists a widespread belief in the literature that three-body interactions are necessary for such a stabilization [96-100].

The information about the stability of the bco and diamond phases is already hidden in the radial distribution function $g(r)$ of a stable liquid with $f=40 \mathrm{arms}$, lying slightly above the domains of stability of these phases. Indeed, this $g(r)$ shows a pronounced peak close to the origin and the fluid state coordination number is about two above the stability domain of the bco and four above that of the diamond. The same is seen in the angle-averaged $g(r)$ 's of the solid phases [38]. A very similar effect was found by Broughton and $\mathrm{Li}$ [101] for the bond-angle dependent StillingerWeber potential [96], which was constructed with the goal of stabilizing the diamond lattice. Here, however, the latter has been stabilized for the first time by means of a simple, radially symmetric pair interaction alone. Moreover, the second freezing observed in experiments on starlike copolymeric micelles $[5,102,103]$, can now be understood as the freezing of the system into the anisotropic bco phase, see figure 6 .

\section{The mean-field character of the star polymer fluid}

The ultrasoft, logarithmic divergence of the star-star potential of equation (3.9) gives rise to some novel features of the macroscopic star polymer solution. On the one hand, it opens up the possibility that for low enough $f$, the system does not 
freeze at any density, a feature unknown for the usual hard or power-law diverging interactions. In this way, the correlation functions of the fluid can be studied at arbitrarily high densities. On the other hand, the ultrasoftness of the interaction renders it integrable in three dimensions, meaning that the integral

$$
\int \mathrm{d}^{3} r v(r)=4 \pi \int_{0}^{\infty} \mathrm{d} r r^{2} v(r)=\tilde{v}(0),
$$

is finite and equal (by definition) to the value of the Fourier transform of the potential $\tilde{v}(k)$ at $k=0$.

Before discussing the ramifications of these properties on the thermodynamics of the star polymer fluid, we point out that recently quite a bit of attention has been focussed on a related but distinct class of fluids, namely the ones whose constituent particles interact by means of positive-definite, purely repulsive potentials that $d o$ not diverge at the origin [104-110]. It was shown that for such systems, a 'meanfield limit' is satisfied [109]: at sufficiently high densities for all temperatures and at sufficiently high temperatures for all densities, the direct correlation function $c(r)$ of the fluid [71] is approximated extremely well by its large- $r$ asymptotic behavior, $c(r) \sim-\beta v(r)$ at all $r$. Hence, for bounded potentials and under the condition of high densities and/or temperatures, we can write at all separations and for all densities:

$$
c(r ; \rho)=-\beta v(r) .
$$

To characterize this whole class of systems, Louis coined the term 'mean-field fluids' [110]. Among the many elegant relations satisfied by the thermodynamic quantities of these models [105], here we single out the quadratic scaling of the excess Helmholtz free energy density with the particle density $\rho$ :

$$
\frac{\beta F_{\mathrm{ex}}(\rho)}{V} \equiv f(\rho)=\frac{\beta \tilde{v}(0)}{2} \rho^{2}
$$

which is a direct consequence of the exact compressibility sum rule [71]:

$$
f^{\prime \prime}(\rho)=-\tilde{c}(k=0 ; \rho)=-4 \pi \int_{0}^{\infty} \mathrm{d} r r^{2} c(r ; \rho)
$$

in conjunction with equation (5.2).

We will argue in what follows that the star polymer fluids satisfy approximately the thermodynamical scaling relation (5.3) although they do not satisfy the more stringent structural relation (5.2). Thereby, we introduce the terminology 'strong mean-field fluids' for those discussed in the preceding paragraph and 'weak meanfield fluids' for those that, like the star polymer fluid, feature an interaction that diverges but only weakly so. The divergence of the interaction is responsible for the impossibility to satisfy the strong mean-field condition of equation (5.2). Indeed, the direct correlation function $c(r)$ has to remain finite at $r=0$, whereas the pair potential diverges. Hence, as shown in figure 7(a), there will always exist a region in the neighborhood of the origin in which equation (5.2) cannot be satisfied. At the 

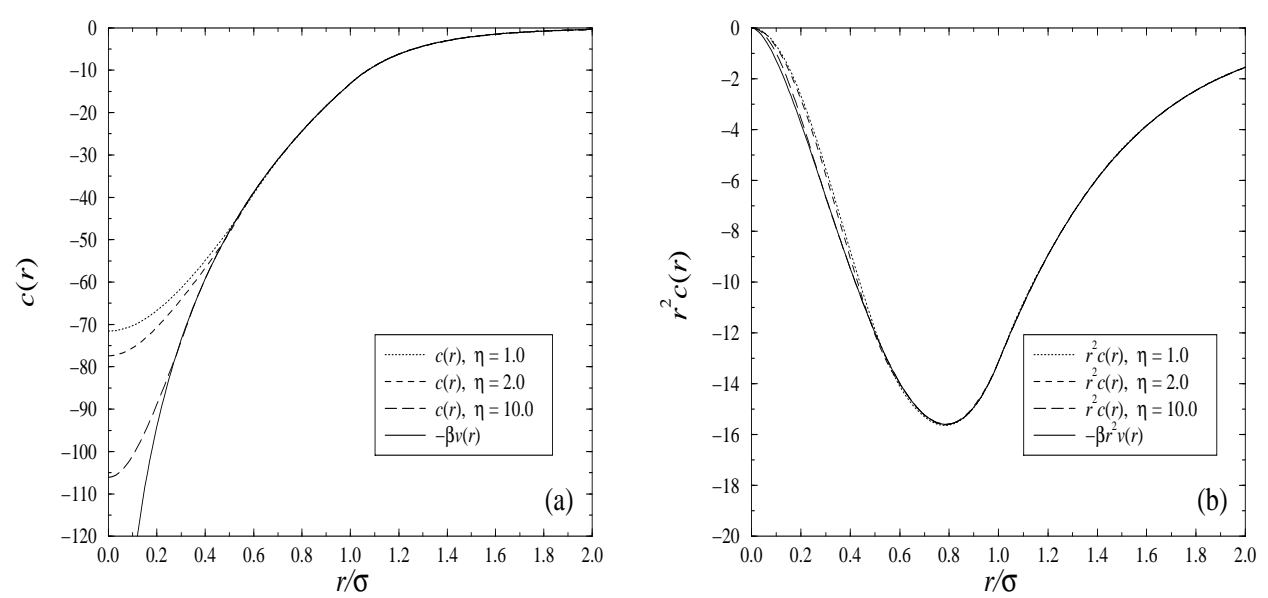

Figure 7. (a) Comparison between the direct correlation function of a $f=32$ star polymer fluid at various densities with the mean-field result, $-\beta v(r)$. (b) Same as in (a) but for the quantities $r^{2} c(r)$ and $-\beta r^{2} v(r)$.

same time, it can be seen in this figure that the extent of this region shrinks with the increasing density, hence the fluid becomes more 'strong mean-field'-like as it gets denser.

The discrepancies between $c(r)$ and $-\beta v(r)$ become innocuous when we turn our attention to the thermodynamics. To obtain the excess Helmholtz free energy, one needs only the integral of $r^{2} c(r)$, see equation (5.4). As demonstrated in figure 7(b), upon multiplication with the geometrical factor $r^{2}$, the deviations of $c(r)$ from $-\beta v(r)$ become suppressed, so that we can write, to a very good approximation:

$$
\int_{0}^{\infty} \mathrm{d} r r^{2} c(r ; \rho) \cong-\int_{0}^{\infty} \mathrm{d} r r^{2} \beta v(r)
$$

Equation (5.5) together with equation (5.4) yield an approximate scaling of the excess free energy of the weak mean-field fluids with density that is identical to that of the strong mean-field fluids, equation (5.3). The accuracy of the approximation for the $f=32$ star fluid is shown in figure 8. The line labeled as exact free energy there was obtained by solving the Rogers-Young closure for the fluid at a wide density range and subsequently utilizing the compressibility sum rule [equation (5.4)] to obtain the excess free energy. Comparisons with simulations [33] have indeed demonstrated that this procedure delivers an essentially exact numerical result.

Clearly, the mean-field approximation improves with the increasing density, since the number of particles effectively interacting with one another grows. The crossover density $\rho_{\times}$above which the quadratic scaling of the free energy holds is $f$-dependent and grows with the increasing $f$. Indeed, the functionality acts as a prefactor that controls the strength of the logarithmic divergence of the potential at the origin. Formally, the mean-field approximation also becomes better at a growing spatial dimension $d$, since the geometrical prefactor $r^{d-1}$ multiplying $c(r)$ and $-\beta v(r)$ suppresses the small- $r$ discrepancies of the two more efficiently. However, we reiterate 


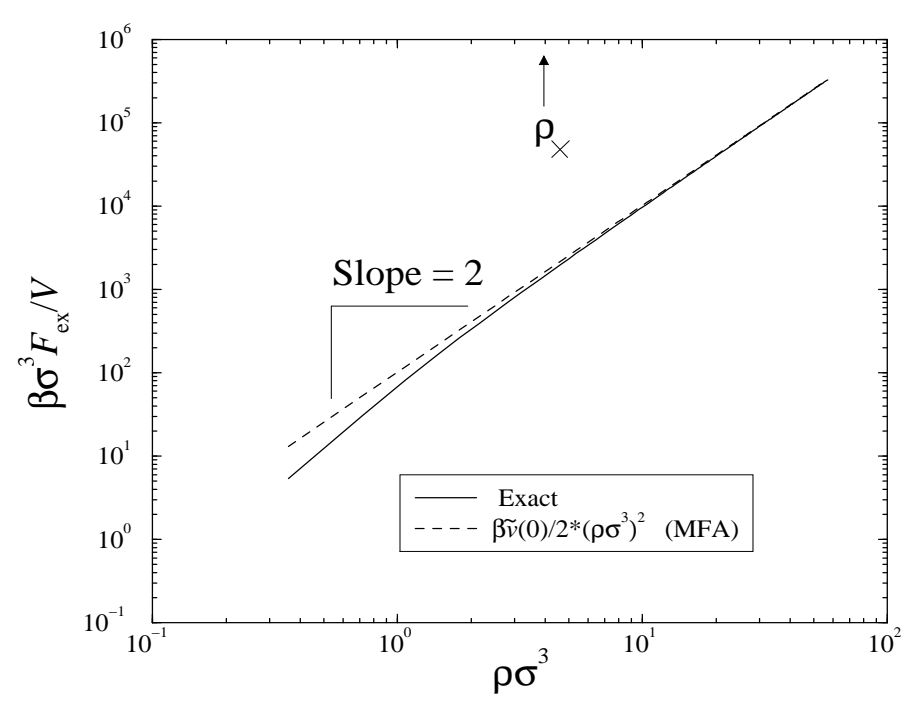

Figure 8. Comparison of the mean-field approximation (dashed line) with the exact result (solid line) concerning the excess free energy density of the $f=32$ star fluid. The slope of the straight line is 2 , indicating the quadratic dependence of the excess free energy density on particle density. The arrow indicates the location of the crossover density $\rho_{\times}$, above which the scaling of equation (5.3) holds with a relative error of less than $10 \%$.

that the mean-field idea holds only for the thermodynamics of weak mean-field fluids: if one naïvely applies the strong mean-field relation, equation (5.2), to weak mean-field fluids, one obtains results for the structure factor $S(k)=[1-\rho \tilde{c}(k)]^{-1}$ that are seriously in error for finite $k$-values. Only at $k=0$ and at sufficiently high densities is it a reasonable approximation to set $S(0)=[1+\rho \beta \tilde{v}(0)]^{-1}$.

\section{Star polymers in gravitational fields}

The mean-field character of star polymer fluids presented in the preceding section manifests itself in the sedimentation profiles of the same. Furthermore, the sedimentation profiles provide an independent check for the bulk phase diagram since the effects stemming from the reentrant melting behavior are prominent. Sedimentation profiles of star polymer solutions were investigated by Dzubiella et al. [111] using MC simulations as well as by applying different theoretical approaches. The MC simulations were carried out in a gravitational field of strength

$$
\alpha=\frac{m g \sigma}{k_{\mathrm{B}} T},
$$

with $m$ denoting the star polymer mass and $g$ denoting the gravitational acceleration. Apart from $\alpha$, the following two thermodynamic variables characterize the system: the arm number $f$ of the star polymers and the number density per unit surface, $\tau$, 
given by the normalization condition of the density profile $\rho(z)$ :

$$
\tau=\int_{0}^{\infty} \rho(z) \mathrm{d} z
$$

The simulations carried out by the authors of [111] revealed the density profiles shown in figure 9. Let us first turn our attention to figures 9a and 9b, where density
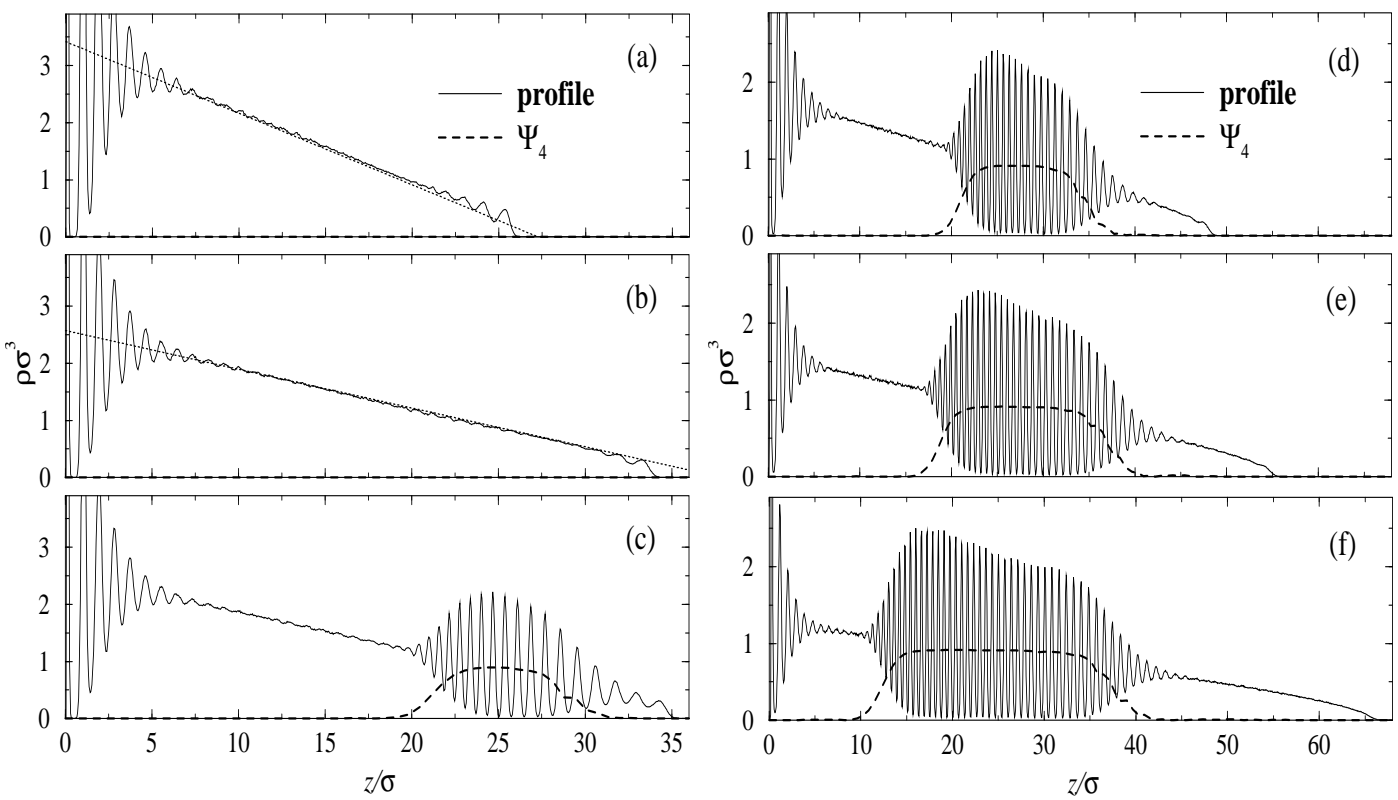

Figure 9. Sedimentation profiles of star polymers for an arm number $f=39$ and a density $\tau \sigma^{2}=48.87$. The gravitational strength $\alpha$ is decreased from (a) to (f) with (a) $\alpha=30.0$, (b) $\alpha=17.0$, (c) $\alpha=16.0$, (d) $\alpha=8.0$, (e) $\alpha=6.0$ and (f) $\alpha=4.0$. In plots (c)-(f) the order parameter $\Psi_{4}$ is also shown (dashed line) using the same $y$-scale as the profiles. In (a) and (b) a straight line the equation of which is derived within the LDA [see equation (6.7)] is superimposed on the plots (dotted line).

profiles for strong gravitational fields $\alpha>\alpha^{*}$ are shown. Beyond the layering on the wall due to packing effects, a fluid regime with the density decaying as a linear function of altitude $z$ can be discerned. At some height $(z \simeq 25 \sigma$ in (a)) the density rapidly decays to zero. At this strong inhomogeneity, oscillations in density with wavelength $\sigma$ can be distinguished in the sedimentation profile, which is smooth elsewhere in the linear regime. These could be reproduced [111] in the framework of the hybrid-weighted-density-approximation (HWDA) density functional theory [112]. By lowering the gravitational strength $\alpha$ further, see figures $9 \mathrm{c}$ to $9 \mathrm{f}$, a critical strength $\alpha^{*}$ in the range $16.0<\alpha^{*}<17.0$ is discovered. Below $\alpha^{*}$ the density profiles qualitatively change, exhibiting strong density oscillations, a clear indication for a crystalline phase. They extend over 10 to 20 star diameters, equivalent to several crystalline layers. The length of the crystal grows as $\alpha$ decreases. Comparing with the bulk phase diagram, it can be presumed that the reentrant melting behavior is mirrored in the density profiles, with a bcc solid intervening in a fluid. 
To complement the indications of the density profiles with an additional check for crystalline order, the local order parameter $\Psi_{4}$ that checks for fourfold symmetry in two dimensions around a given particle was calculated. It is defined as

$$
\Psi_{4}(z)=\left|\left\langle\frac{1}{4 N_{\mathrm{l}}} \sum_{j=1}^{N_{1}} \sum_{<k>} \mathrm{e}^{4 \mathrm{i} \phi_{j k}}\right\rangle\right|,
$$

where the $k$-sum includes the four nearest neighbors of the given particle and the $j$-sum extends over $N_{1}$ particles in the corresponding layer. The angular brackets indicate a canonical ensemble average. $\phi_{j k}$ is the polar angle of the interparticle distance vector with respect to a fixed reference frame. For ideal fourfold symmetry, i.e., for a particle contained in a bcc-solid layer, $\Psi_{4}=1$. For practical purposes, values of $\Psi_{4}>0.8$ are taken to be conclusive evidence for a crystalline phase with fourfold-in-layer-symmetry. Thus, obviously, a bcc solid is stable and the sedimentation profiles present a confirmation of the bulk phase diagram: the gravitational field induces a scan of all densities as a function of the height $z$, thereby mapping the freezing/reentrant melting succession on the height and producing a solid sheet at intermediate heights intercalated in a fluid at lower and at higher elevations.

The linear dependence of the density profile on $z$, on the other hand, is a conclusive evidence for the star polymer fluids belonging to the class of "weak mean-field fluids', see equation (5.3), as we demonstrate below. We work in the grand canonical ensemble and introduce a variational grand potential per unit area, $\tilde{\Sigma}(T, \mu ;[\rho(z)])$, which is a functional of the density profile and a function of the chemical potential $\mu$ and temperature $T$. Introducing the ideal and excess per unit area contributions to the intrinsic Helmholtz free energy of the system, $F_{\text {id }}[\rho(z)]$ and $F_{\text {ex }}[\rho(z)]$ respectively, we find that in the local density approximation, the expression for $\tilde{\Sigma}(T, \mu ;[\rho(z)])$ reads as:

$$
\begin{aligned}
\tilde{\Sigma}(T, \mu,[\rho(z)])= & F_{\mathrm{id}}[\rho(z)]+F_{\text {ex }}[\rho(z)]+\int \mathrm{d} z \Phi_{\text {ext }}(z) \rho(z)-\mu \int \mathrm{d} z \rho(z) \\
= & k_{\mathrm{B}} T \int_{0}^{\infty} \mathrm{d} z \rho(z)\left[\ln \left(\rho(z) \lambda^{3}\right)-1\right] \\
& +\int_{0}^{\infty} \mathrm{d} z[f(\rho(z))+(m g z-\mu) \rho(z)]
\end{aligned}
$$

where $\lambda=\sqrt{h^{2} / 2 \pi m k_{\mathrm{B}} T}$ is the thermal de Broglie wavelength and $f(\rho(z))$ is the Helmholtz free energy density of the bulk fluid. The minimization of $\tilde{\Sigma}$ with respect to $\rho(z)$ yields the equilibrium profile $\rho_{0}(z)$; the value of the functional at equilibrium, $\tilde{\Sigma}\left(T, \mu,\left[\rho_{0}(z)\right]\right)$ is then the Gibbs free energy per unit area, $\Sigma(T, \mu)$ of the system. Setting $\delta \tilde{\Sigma}(T, \mu,[\rho(z)]) /\left.\delta \rho(z)\right|_{\rho_{0}(z)}=0$ in equation (6.4), leads to:

$$
k_{\mathrm{B}} T \ln \left[\rho_{0}(z) \sigma^{3}\right]+f^{\prime}\left(\rho_{0}(z)\right)=\mu^{\prime}-m g z,
$$

where $f^{\prime}(x)$ denotes the derivative of $f(x)$ and $\mu^{\prime}=\mu-3 \ln (\lambda / \sigma)$ is a shifted chemical potential. 
At this point, we resort to the the property equation (5.3), which, together with the use of the dimensionless variables $x \equiv z / \sigma, \bar{\rho}(x) \equiv \rho(z) \sigma^{3}, B \equiv \beta \tilde{v}(0) / \sigma^{3}$, $\bar{\mu} \equiv \beta \mu^{\prime}$ and introducing equation (5.3) into equation (6.5), yields the equilibrium profile through:

$$
\ln \left[\bar{\rho}_{0}(x)\right]+B \bar{\rho}_{0}(x)=\bar{\mu}-\alpha x
$$

For star functionality $f=39$, we obtain $B=250.4$ [see equation (3.9)]. Hence, the second term in the lhs of equation (6.6) above dominates over the logarithmic term for densities $\bar{\rho}(x) \gtrsim 0.10$. As far as almost the entire simulation density profile fulfills this condition, we finally omit the logarithmic term from equation (6.6) above. Using the normalization condition $\int_{0}^{\bar{\mu} / \alpha} \mathrm{d} x \bar{\rho}_{0}(x)=\tau \sigma^{2} \equiv \bar{\tau}$, the chemical potential $\bar{\mu}$ can be evaluated. Finally, we thereby obtain a linear density profile:

$$
\bar{\rho}_{0}(x)= \begin{cases}0 & \text { for } x<0 \\ \sqrt{\frac{2 \alpha \bar{\tau}}{B}}-\frac{\alpha}{B} x & \text { for } 0<x<\sqrt{\frac{2 B \bar{\tau}}{\alpha}} \\ 0 & \text { for } \sqrt{\frac{2 B \bar{\tau}}{\alpha}}<x .\end{cases}
$$

In figures $9 \mathrm{a}$ and $9 \mathrm{~b}$, this prediction, which crucially depends on the mean-field approximation used, is checked against simulation. Excellent agreement is found, thereby confirming again the mean-field character of star polymer solutions. Moreover, a phenomenological Landau theory revealed a host of scaling relations satisfied by the thickness of the intercalating solid layer, which have been explicitly verified by Monte Carlo simulations [111].

\section{Summary and concluding remarks}

In this paper, we have presented a concise review of a recent progress achieved in understanding the properties of star polymer solutions in terms of effective interactions between the stars' centers. This constitutes a coarse-grained description of the system, in which all degrees of freedom at atomic length scales $(\sim 1 \AA)$ have been traced out and hence they drop out of the picture. In our mesoscopic description, the only relevant length scales are the size of the stars $\sigma$ (typically of the order of $100 \AA$ or more) and the structural length scale $a=\rho^{-1 / 3}$ set by the star density. It has been demonstrated that this approach is indeed fruitful. By integrating out the details of the monomers at the one- and two-star level, an enormous simplification of the many-star problem is achieved: the total number of degrees of freedom is reduced from $N_{s} \times N \times f$ to $N_{s}$, i.e., the number of stars in the system. Though the effective interactions presented here have been derived strictly for star polymers with a vanishingly small core, they should remain approximately valid for macromolecular aggregates with a core-brush (shell) structure, such as sterically stabilized colloids and micelles, provided that the ratio of core size to brush height is sufficiently small. 


\section{Acknowledgements}

We thank J.Dzubiella, A.Jusufi, C. von Ferber, A.Lang, H.Löwen, and M.Watzlawek for fruitful collaboration and J.Dzubiella for a critical reading of the manuscript. This work has been supported by the Deutsche Forschungsgemeinschaft through the SFB 237.

\section{References}

1. Hunter R.J. Foundations of Colloid Science. Vol. I. Oxford, Clarendon Press, 1986.

2. Likos C.N. // Phys. Rep., 2001, vol. 348, p. 267.

3. Gast A.P., Russel W.B. // Physics Today, 1998, vol. 51, p. 24.

4. Gast A.P. // Langmuir, 1996, vol. 12, p. 4060.

5. Gast A.P. // Curr. Opinion Coll. Int. Sci., 1997, vol. 2, p. 258.

6. Grest G.S., Fetters L.J., Huang J.S., Richter D. // Adv. Chem. Phys., 1996, vol. XCIV, p. 67.

7. Burchard W. // Adv. Polym. Sci., 1999, vol. 143, p. 113.

8. Freire J.J., Pla J., Rey A., Prats R. // Macromolecules, 1986, vol. 19, p. 452.

9. Zhou L.-L., Roovers J. // Macromolecules, 1993, vol. 26, p. 963.

10. Roovers J., Zhou L.-L., Toporowski P.M., van der Zwan M., Iatrou H., Hadjichristidis N. // Macromolecules, 1993, vol. 26, p. 4324.

11. Dozier W.D., Huang J.S., Fetters L.J. // Macromolecules, 1991, vol. 24, p. 2810.

12. Mezei F. - In: Neutron Spin Echo. ed. by Mezei F., Berlin, Springer Verlag, 1979.

13. Solc K., Stockmayer W.H. // J. Chem. Phys., 1971, vol. 54, p. 2756.

14. Solc K. // J. Chem. Phys., 1971, vol. 55, p. 2335.

15. Tanaka G., Mattice W.L. // Macromol. Theory Simul., 1996, vol. 5, p. 499.

16. Solc K. // Macromolecules, 1973, vol. 6, p. 378.

17. Solc K. // Macromolecules, 1980, vol. 13, p. 506.

18. Batoulis J., Kremer K. // Macromolecules, 1989, vol. 22, p. 4277.

19. Zifferer G. // J. Chem. Phys., 1995, vol. 102, p. 3720.

20. Zifferer G. // Macromol. Theory Simul., 1997, vol. 6, p. 381.

21. Zifferer G. // J. Chem. Phys., 1999, vol. 110, p. 4668.

22. Forni A., Ganazzoli F., Vacatello M. // Macromolecules, 1997, vol. 30, p. 4737.

23. Sikorski A., Romiszowski P. // J. Chem. Phys., 1998, vol. 109, p. 6169.

24. Witten T.A., Pincus P.A., Cates M.E. // Europhys. Lett., 1986, vol. 2, p. 137.

25. Witten T.A., Pincus P.A. // Macromolecules, 1986, vol. 19, p. 2509.

26. Richter D., Jucknischke O., Willner L., Fetters L.J., Lin M., Huang J.S., Roovers J., Toporowski C., Zhou L.L. // J. Physique IV Suppl., 1993, vol. 3, p. 3.

27. Roovers J. // Macromol. Symp., 1997, vol. 121, p. 89.

28. Adam M., Fetters L.J., Graessley W.W., Witten T.A. // Macromolecules, 1991, vol. 25, p. 2434.

29. Willner L., Jucknischke O., Richter D., Farago B., Fetters L.J., Huang J.S. // Europhys. Lett., 1992, vol. 19, p. 297.

30. Willner L., Jucknischke O., Richter D., Roovers J., Zhou L.-L., Toporowski P.M., Fetters L.J., Huang J.S., Lin M.Y., Hadjichristidis N. // Macromolecules, 1994, vol. 27, p. 3821 . 
31. Likos C.N., Löwen H., Watzlawek M., Abbas B., Jucknischke O., Allgaier J., Richter D. // Phys. Rev. Lett., 1998, vol. 80, p. 4450.

32. Likos C.N., Löwen H., Poppe A., Willner L., Roovers J., Cubitt B., Richter D. // Phys. Rev. E, 1998, vol. 58, p. 6299.

33. Watzlawek M., Löwen H., Likos C.N. // J. Phys.: Condens. Matter, 1998, vol. 10, p. 8189 .

34. Stellbrink J., Abbas B., Allgaier J., Monkenbusch M., Richter D., Likos C.N., Löwen H., Watzlawek M. // Progr. Coll. Polym. Sci., 1998, vol. 110, p. 25.

35. Watzlawek M., Likos C.N., Löwen H. // Phys. Rev. Lett., 1999, vol. 82, p. 5289.

36. von Ferber C., Jusufi A., Likos C.N., Löwen H., Watzlawek M. // Eur. Phys. J. E, 2000 , vol. 2 , p. 311.

37. von Ferber C., Jusufi A., Watzlawek M., Likos C.N., Löwen H. // Phys. Rev. E, 2000, vol. 62, p. 6949.

38. Watzlawek M. Phase Behavior of Star Polymers. Aachen, Shaker-Verlag, 2000.

39. Roovers J. // Macromolecules, 1994, vol. 27, p. 5359.

40. Seghrouchni R., Petekidis G., Vlassopoulos D., Fytas G., Semenov A.N., Roovers J., Fleischer G. // Europhys. Lett., 1998, vol. 42, p. 271.

41. Stellbrink J., Allgaier J., Richter D. // Phys. Rev. E, 1997, vol. 56, p. R3772.

42. Vlassopoulos D., Pakula T., Fytas G., Roovers J., Karatasos K., Hadjichristidis N. // Europhys. Lett., 1997, vol. 39, p. 617.

43. Nommensen P.A., Duits M.H.G., Lopulissa J.S., van den Ende D., Mellema J. // Progr. Coll. Polym. Sci., 1998, vol. 110, p. 144.

44. Fleischer G., Fytas G., Vlassopoulos D., Roovers J., Hadjichristidis N. // Physica A, 2000, vol. 280, p. 266.

45. Semenov A.N., Vlassopoulos D., Fytas G., Vlachos G., Fleischer G., Roovers J. // Langmuir, 1999, vol. 15, p. 358.

46. Vlassopoulos D., Fytas G., Roovers J., Pakula T., Fleischer G. // Faraday Discuss., 1999, vol. 112, p. 225.

47. Kapnistos M., Semenov A.N., Vlassopoulos D., Roovers J. // J. Chem. Phys., 1989, vol. 111, p. 1753.

48. Vlassopoulos D., Pakula T., Fytas G., Pitsikalis M., Hadjichristidis N. // J. Chem. Phys., 1999, vol. 111, p. 1760.

49. Grest G.S., Kremer K., Witten T.A. // Macromolecules, 1987, vol. 20, p. 1376.

50. Jusufi A. Diploma thesis. Düsseldorf, Heinrich-Heine-Universität, 1998.

51. Daoud M., Cotton J.P. // J. Phys. (Paris), 1982, vol. 43, p. 531.

52. de Gennes P.G. Scaling Concepts in Polymer Physics. Ithaca, Cornell University Press, 1979.

53. Vilgis T.A. // Phys. Rep., 2000, vol. 336, p. 167.

54. Jusufi A., Dzubiella J., Likos C.N., von Ferber C., Löwen H. // J. Phys.: Condens. Matter, 2001, vol. 13, p. 6177.

55. Dzubiella J., Jusufi A., Likos C.N., von Ferber C., Löwen H., Stellbrink J., Allgaier J., Richter D., Schofield A.B., Smith P.A., Poon W.C.K., Pusey P.N. // Phys. Rev. E, 2001, vol. 64, p. 010401(R).

56. Batoulis J., Kremer K. // Europhys. Lett., 1988, vol. 7, p. 683.

57. Grest G.S. // Macromolecules, 1994, vol. 27, p. 3493.

58. Shida K., Ohno K., Kimura M., Kawazoe Y., Nakamura Y. // Macromolecules, 1998, vol. 31, p. 2343. 
59. Molina L.A., Freire J.J. // Macromolecules, 1999, vol. 32, p. 499.

60. Grest G.S., Murat M. - In: Monte Carlo and Molecular Dynamics Simulations in Polymer Science. ed. by Binder K., New York, Oxford University Press, 1995.

61. Forni A., Ganazzoli F., Vacatello M. // Macromolecules, 1996, vol. 29, p. 2994.

62. Rubio A.M., Freire J.J. // Macromolecules, 1996, vol. 29, p. 6946.

63. Jusufi A., Watzlawek M., Löwen H. // Macromolecules, 1999, vol. 32, p. 4470.

64. Su S.-J., Denny M.S., Kovac J. // Macromolecules, 1991, vol. 24, p. 917.

65. Su S.-J., Kovac J. // J. Chem. Phys., 1992, vol. 96, p. 3931.

66. Toral R., Chakrabarti A. // Phys. Rev. E, 1993, vol. 47, p. 4240.

67. Barrett A.J., Tremain D.L. // Macromolecules, 1987, vol. 20, p. 1687.

68. Rey A., Freire J.J., de la Torre J.G. // Macromolecules, 1987, vol. 20, p. 342.

69. Gratter O., Kratky O. Small Angle X-Ray Scattering. New York, Academic Press, 1982.

70. Higgins J.S., Benoît H.C. Polymers and Neutron Scattering. Oxford, Clarendon Press, 1994.

71. Hansen J.-P., McDonald I.R. Theory of Simple Liquids. 2nd ed. London, Academic Press, 1986.

72. des Cloizeaux J. // J. Phys. (Paris), 1980, vol. 41, p. 223.

73. von Ferber C., Holovatch Yu. // Theor. Math. Phys., 1996, vol. 109, p. 34.

74. von Ferber C., Holovatch Yu. // Europhys. Lett., 1997, vol. 39, p. 31.

75. von Ferber C., Holovatch Yu. // Phys. Rev. E, 1997, vol. 56, p. 6370.

76. von Ferber C., Holovatch Yu., Jusufi A., Likos C.N., Löwen H., Watzlawek M. // J. Mol. Liquids, 2001, vol. 93, p. 151.

77. Bolhuis P.G., Louis A.A., Hansen J.-P., Meier E.J. // J. Chem. Phys., 2001, vol. 114, p. 4296.

78. Ohno K., Shida K., Kimura M., Kawazoe Y. // Macromolecules, 1996, vol. 29, p. 2269.

79. Rubio A.M., Freire J.J. // Comp. Theor. Polymer Sci., 2000, vol. 10, p. 89.

80. Shida K., Ohno K., Kimura M., Kawazoe Y. // Macromolecules, 2000, vol. 33, p. 7655 .

81. Striolo A., Prausnitz J.M., Bertucco A. // Macromolecules, 2000, vol. 33, p. 9583.

82. Stellbrink J., Allgaier J., Monkenbusch M., Richter D., Lang A., Likos C.N., Watzlawek M., Löwen H., Ehlers G., Schleger P. // Progr. Coll. Polym. Sci., 2000, vol. 115, p. 88.

83. Jucknischke O. Ph.D. thesis. Münster, Westfälische Wilhelms-Universität, 1995.

84. Abbas B. Diploma thesis. Münster, Westfälische-Wilhelms Universität, 1996.

85. Buitenhuis J., Förster S. // J. Chem. Phys., 1997, vol. 107, p. 262.

86. Dijkstra M., van Roij R., Evans R. // Phys. Rev. E, 1999, vol. 59, p. 5744.

87. Rogers F.A., Young D.A. // Phys. Rev. A, 1984, vol. 30, p. 999.

88. Frenkel D., Smit B. Understanding Molecular Simulation. San Diego, Academic Press, 1996.

89. Allen M.P., Tildesley D.J. Computer Simulation of Liquids. Oxford, Clarendon Press, 1987.

90. Ashcroft N.W., Mermin N.D. Solid State Physics. Philadelphia, Holt Saunders, 1976.

91. Sirota E.B., Ou-Yang H.D., Sinha S.K., Chaikin P.M. // Phys. Rev. Lett., 1989, vol. 62 , p. 1524.

92. Kremer K., Robbins M.O., Grest G.S. // Phys. Rev. Lett., 1986, vol. 57, p. 2694. 
93. Robbins M.O., Kremer K., Grest G.S. // J. Chem. Phys., 1988, vol. 88, p. 3286.

94. McConnell G.A., Gast A.P., Huang J.S., Smith S.D. // Phys. Rev. Lett., 1993, vol. 71, p. 2102.

95. McConnell G.A., Lin E.K., Gast A.P., Huang J.S., Lin M.Y., Smith S.D. // Faraday Discuss. Chem. Soc., 1994, vol. 98, p. 121.

96. Stillinger F.H., Weber T.A. // Phys. Rev. B, 1985, vol. 31, p. 5262.

97. Tersoff J. // Phys. Rev. Lett., 1986, vol. 56, p. 632.

98. Biswas R., Hamann D.R. // Phys. Rev. Lett., 1985, vol. 55, p. 2001.

99. Phillpot R.S., Lutsko J.F., Wolf D., Yip S. // Phys. Rev. B, 1989, vol. 40, p. 2831.

100. Likos C.N., Ashcroft N.W. // Phys. Rev. E, 1995, vol. 52, p. 5714.

101. Broughton J.Q., Li X.P. // Phys. Rev. B, 1987, vol. 35, p. 9120.

102. McConnell G.A., Gast A.P. // Phys. Rev. E, 1996, vol. 54, p. 5447.

103. McConnell G.A., Gast A.P. // Macromolecules, 1997, vol. 30, p. 435.

104. Likos C.N., Watzlawek M., Löwen H. // Phys. Rev. E, 1998, vol. 58, p. 3135.

105. Lang A., Likos C.N., Watzlawek M., Löwen H. // J. Phys.: Condens. Matter, 2000, vol. 12 , p. 5087.

106. Louis A.A., Bolhuis P.G., Hansen J.-P., Meier E.J. // Phys. Rev. Lett., 2000, vol. 85, p. 2522 .

107. Louis A.A., Bolhuis P.G., Hansen J.-P. // Phys. Rev. E, 2000, vol. 62, p. 7961.

108. Likos C.N., Schmidt M., Löwen H., Ballauff M., Pötschke D., Lindner P. // Macromolecules, 2000, vol. 34, p. 2914.

109. Likos C.N., Lang A., Watzlawek M., Löwen H., Phys. Rev. E, 2001, vol. 63, p. 031206.

110. Louis A.A. // Philos. Trans. Ros. Soc. A, 2001, vol. 359, p. 939.

111. Dzubiella J., Harreis H.M., Likos C.N., Löwen H. // Phys. Rev. E, 2001, vol. 64, p. 011405.

112. Leidl R., Wagner H. // J. Chem. Phys., 1993, vol. 98, p. 4142. 


\title{
Зіркові полімери: від конформацій через взаємодії до фазових діаграм
}

\author{
К.Н.Лікос, Г.М.Харрайс \\ Інститут теоретичної фізики II, Університет Гайнріха Гайне, \\ Університатштрасе 1, D-40225 Дюссельдорф, Німеччина \\ Отримано 9 жовтня 2001 p.
}

\begin{abstract}
Здійснено огляд недавніх досягнень у теоретичному описі взаємодій, кореляцій і фазової поведінки концентрованих розчинів зіркових полімерів, просторово стійких колоїдів і міцел. Ми покажемо, що теоретично передбачена надм'яка логарифмічно розбіжна ефективна взаємодія між центрами зірок, що була підтверджена SANSекспериментами і комп'ютерними симуляціями, потрапляє в множину незвичних явищ, які спостерігаються в таких системах. Сюди відносяться аномальні структурні фактори, поведінка зворотнього плавлення, множини екзотичних кристалічних фаз. Також обговорено узагальнення на випадок полідисперсних зірок і роль сил багатьох тіл. Представлено особливу поведінку типу “середнього поля" плинів зіркових полімерів і показано, що вона проявляється у формі і структурі профілів осаджування цих систем.
\end{abstract}

Ключові слова: полімери, колоїди, теорія рідкого стану, фазові переходи.

PACS: $82.70 . \mathrm{Dd}, 61.25 . \mathrm{Hq}, 61.20 .-p, 64.70 . \mathrm{Dv}$ 